\title{
LA ESCUELA DE SALAMANCA SEGÚN JOSÉ BARRIENTOS: ORIGEN, DIFUSIÓN E IMPACTO INTELECTUAL EN EUROPA (SIGLOS XVI AL XVIII)
}

\author{
THE SCHOOL OF SALAMANCA ACCORDING TO JOSÉ BARRIEN- \\ TOS: ITS ORIGIN, DISSEMINATION AND \\ INTELLECTUAL IMPACT IN EUROPE (XVI-XVIII CENTURIES)
}

\author{
GERMÁN SCALZO \\ Universidad Panamericana \\ ANTONIO MORENO ALMÁrCEGUI \\ Universidad de Navarra
}

Recibido: 29/07/2019 Aceptado: 23/09/2019

\section{RESUMEN}

Existe en la actualidad un renovado interés por la Escuela de Salamanca, así como una gran diversidad de opiniones acerca de su definición y alcance. Este artículo realiza un análisis sistemático de los autores propuestos por José Barrientos en su Repertorio de moral económica (1536-1670): la Escuela de Salamanca y su proyección (EUNSA, 2011), pertenecientes a los siglos XVI a XVIII. A partir del estudio de la información provista por Barrientos -los años de edición de las obras, lugares de publicación, la variación en los autores citados, las órdenes religiosas a las que pertenecen y las temáticas centrales tratadas- se pretende contribuir a la comprensión del auge y decadencia de la escuela, así como su importancia en la historia económica y política de Occidente. Encontramos en ella el primer intento de configurar, desde la tradición bajomedieval y el humanismo renacentista, un pensamiento especializado y sistemático en torno a los problemas económicos y sociales. 
Palabras clave: contrato, don, Escuela de Salamanca, José Barrientos, moral económica.

\section{ABSTRACT}

A diversity of opinions concerning what the School of Salamanca is flourish in contemporary academic literature, and interest in its study has notably increased. This paper offers a systematic analysis of the sixteenth to eighteenth-century authors that José Barrientos presents in his work Repertorio de moral económica (1536-1670): la Escuela de Salamanca y su proyección (EUNSA, 2011). A study of the information that Barrientos provides -including years of edition of the different works, the places of publication, variations in the cited authors, the religious orders to which their authors belong and the central themes discussed-aims to show the rise and decline of the School, and its importance for the West's economic and political history. It concludes that the School of Salamanca's efforts were the first attempt to configure specialized and systematic thought about economic and social problems starting from the medieval tradition and up to Renaissance humanism.

Keywords: business ethics, insurances, Leibniz, providence, usury.

\section{INTRODUCCIÓN}

En los últimos años ha surgido un renovado interés por la economía premoderna, y en especial por la Escuela de Salamanca ${ }^{1}$. El presente estudio es un análisis sistemático de la información aportada por Barrientos en su obra Repertorio de Moral Económica (1536-1670): la Escuela de Salamanca y su Proyección ${ }^{2}$. José Barrientos García es profesor titular de Historia de la Filosofía en la Universidad de Salamanca y, a través de su obra, tiene acreditado un amplio y profundo conocimiento de los autores y las fuentes de la escuela salmantina. Es autor de diversos libros acerca del pensamiento salmantino de los siglos XVI y XVII (como "Lucha por el poder y la libertad de enseñanza en Salamanca" (1990), "La Escuela de Salamanca: desarrollo y caracteres" (1995) y "Fray Luis

1 Véase, por ejemplo, León Gómez Rivas, "Business Ethics and The History of Economics in Spain «The School of Salamanca: A Bibliography»", Journal of Business Ethics 22 (1999): 191-202 y Belda Plans, J. "Hacia una noción crítica de la Escuela de Salamanca", Scripta Theologica, 31 (1999/2): $367-411$.

2 José Barrientos García, Repertorio de moral económica (1536-1570): La Escuela de Salamanca y su proyección (Pamplona: EUNSA 2011). 
de León y la Universidad de Salamanca" (1996)), así como de diversos monográficos y ediciones. Esta obra que aquí se analiza representa el culmen de una vida dedicada al estudio sistemático de las cuestiones de moral económica tratadas por la Escuela de Salamanca, enriquecida por el acceso directo del profesor salmantino a las fuentes originales. Es verdad que los criterios de Barrientos para la selección de autores pueden ser discutibles; pero también es verdad que hasta el momento no había habido una obra que reuniera información de manera tan amplia y sistemática -apoyada en el estudio de 95 autores- sobre la Escuela de Salamanca. Por eso, aunque no hay acuerdo total sobre los límites y dimensiones de la escuela de Salamanca, en este trabajo aceptamos como razonablemente representativa la muestra de Barrientos sobre la Escuela tal como se presenta en la obra citada.

El interés de la obra de Barrientos es triple. Primero, al no limitarse el autor a los autores salmantinos y extender el estudio a 'su proyección', el análisis de la obra permite valorar la difusión de Escuela de Salamanca en el espacio -la Europa católica surcada por la Reforma y el Concilio de Trento-y en el tiempo -segunda mitad del siglo XVI, el XVII y crisis del XVIII-, aspecto todavía no bien conocido y que nos parece de un extraordinario interés. Así, la obra de Barrientos descubre la dimensión 'europea' de la escuela Salmantina.

El segundo interés de la obra tiene que ver con el objeto temático: la moral económica. Como se sabe, en estos siglos la ciencia económica aún no constituye un saber especializado y los autores de la escuela tratan de estos temas dentro del tratado más general sobre la justicia. Así, Barrientos ha tenido que hacer una tarea ingente, minuciosa y paciente de exploración en los textos originales -que conoce de primera mano-, extrayendo aquello que podría considerarse 'económico'. Lógicamente, el valor de los resultados depende de su criterio (y el autor no aclara de modo explícito el criterio seguido), pero es tal el dominio del autor de las obras de los autores seleccionados, así como el volumen de referencias recopilado, y tan detallado el análisis de las citas y autores referenciados $^{3}$ que, aunque con errores y lagunas, los resultados más importantes nos parecen razonablemente sólidos. Por otro lado, al conocerse y citarse entre sí, estos autores tienden a repetir los mismos temas, lo que favorece la selección sistemática que encontramos. Por supuesto, la metodología aplicada a esos datos es responsabilidad nuestra.

3 Para hacerse una idea del grado de detalle con el que trabaja Barrientos, fruto del análisis de las obras recoge un total de 140.929 citas, de las cuales 64.183 describen detalladamente a cada uno de los autores citados. 
Como hemos dicho, Barrientos en su obra distingue entre aquellos teólogos que pertenecen a la Escuela de Salamanca en sentido estricto y aquéllos que forman parte de su proyección ${ }^{4}$. Así, el autor no se limita a un estudio estricto de los maestros salmantinos, sino que va más allá, extendiéndose a todos aquellos autores influenciados más o menos directamente por éstos. Esta interpretación amplia de la escuela salmantina posee una gran ventaja, ya que permite hacer un estudio del impacto en la Europa intelectual de los siglos XVI, XVII y XVIII de las ideas de los autores salmantinos, que es el objeto principal de este artículo. Asimismo, de modo indirecto, y esta es la tercera razón para el análisis de la obra, este estudio permitirá reconstruir la expansión, auge y crisis del tomismo a lo largo de la Edad Moderna, aspecto totalmente desconocido hasta ahora, al menos en lo que se refiere al tomismo explicado en las aulas de las universidades españolas y-gracias a su difusión jesuita- europeas.

¿Quiénes forman la Escuela de Salamanca para nuestro autor? Para Barrientos, los miembros de la Escuela de Salamanca en sentido estricto son los que enseñaron a Santo Tomás en las aulas de la Escuela de Salamanca siguiendo para ello las directrices marcadas por el colegio dominico de San Esteban. En la selección de autores, Barrientos considera que la Escuela de Salamanca es una escuela teológica, fundada por Francisco de Vitoria, que se basa primordialmente en la doctrina de Santo Tomás, aunque éste no siempre fuera objeto de las mismas interpretaciones. Esta fórmula permite definir la Escuela de Salamanca con dos simples vocablos: teológica y tomista ${ }^{5}$.

Al hablar de la Escuela de Salamanca como tomista, no hay que asociarlo con el tomismo concreto de determinados autores, sino como una realidad cambiante a lo largo de los años. En efecto, el tomismo de Vitoria no era rígido, sino que era muy abierto a las ideas humanistas: "Vitoria fue un adelantado en lo que tuvo de renacentista y humanista, [porque] estuvo a la altura de los tiempos"6. Melchor Cano y Domingo de Soto supieron dar continuidad a dicha concepción, que sufre posteriormente una inflexión, hasta llegar a Domingo de Báñez, que era un tomista "puro e integral"”. De ahí que, como criterio identificador de los autores de la Escuela de Salamanca, se resalte la adhesión a las pautas del colegio de San Esteban, que no siempre fueron homogéneas a lo largo de los años, como se ha mencionado antes. La discrepancia con las doctrinas que emanaban

\footnotetext{
4 José Barrientos García, Repertorio de moral económica ..., 84.

5 Ibidem, 20.

6 Vicente Muñoz Delgado, Lógica ciencia y humanismo en la renovación teológica de Vitoria y Cano (Madrid: Instituto Francisco Suárez del C.S.I.C, 1980), 51.

7 Vicente Beltrán de Heredia, "Accidentada y efímera aparición del nominalismo en Salamanca", en Miscelánea de Beltrán de Heredia: Colección de artículos sobre la historia de la teología española (Salamanca: [Editorial], 1972), 525.
} 
de dicho colegio son por ende un criterio excluyente, lo que implica que figuras tan ilustres como por ejemplo Fray Luis de León (considerado una víctima ${ }^{8}$ de la Escuela de Salamanca) queden al margen.

Por otra parte, el que para Barrientos la Escuela de Salamanca además de tomista sea también teológica, excluye de este estudio a los docentes de las facultades jurídicas de la Universidad de Salamanca (Cánones y Leyes) ${ }^{9}$ a pesar de las posibles coincidencias con los teólogos en cuanto a los temas y preocupaciones tratadas.

Los miembros de la Proyección de la Escuela de Salamanca, por su parte, son aquellos que citan en sus obras escritas y publicadas a los miembros de la Escuela de Salamanca en sentido estricto, independientemente del volumen de citas o de su adhesión o no a las doctrinas de éstos. Como es lógico, quedan excluidos de este análisis aquellos autores que no citan a los miembros de la Escuela en sentido estricto, así como las obras inéditas de los que sí lo hacen, por obvios motivos de índole práctica ${ }^{10}$.

En virtud de estos criterios, Barrientos elabora una lista de los maestros que conforman el núcleo duro de la Escuela de Salamanca: Francisco de Vitoria, Melchor Cano, Mancio de Corpus Christi, Bartolomé de Medina, Pedro de Aragón, Domingo Báñez, Pedro de Ledesma, Basilio Ponce de León y Francisco de Arujo. El resto de los autores que analiza (85), dan lugar a la Proyección de la Escuela de Salamanca, y tienen diversas nacionalidades (hispánicos, italianos, belgas, franceses, holandeses, ingleses y alemanes) y pertenecen a distintas órdenes religiosas (franciscanos, dominicos, jesuitas, agustinos, teatinos, cistercienses).

El lapso temporal del estudio realizado empieza con el acceso de Francisco de Vitoria a la cátedra de Prima de Teología de la Universidad de Salamanca en el año 1526, que marca el inicio de la Escuela de Salamanca en sentido estricto. El final de ésta vendrá marcado por la última edición de los miembros de la Proyección de la Escuela de Salamanca ya en el siglo XVII.

El presente trabajo, a través de un análisis sistemático de las ediciones, lugares de publicación, citas y temas de los diversos autores que se abordan en dicha obra, tanto hispánicos como extranjeros, pertenecientes a los siglos XVI, XVII y XVIII, pretende aportar evidencia empírica sobre la evolución de dicha escuela en torno a las preocupaciones sociales de su tiempo, con el fin de obtener una visión completa de la evolución del pensamiento económico a lo largo de

8 José Barrientos García, Fray Luis de León y la Universidad de Salamanca ([Ciudad]: El Escorial 1996), 439-569.

9 José Barrientos García, Repertorio de moral económica ..., 84.

10 Ibidem, 201. 
más de tres siglos y de contribuir al debate sobre los elementos definidores y el alcance de la Escuela de Salamanca ${ }^{11}$.

\section{METODOLOGÍA}

La metodología para este estudio será esencialmente cuantitativa: se analiza un inventario detallado de los datos recopilados por Barrientos sobre los 95 autores analizados en su obra. Se proponen tres estudios concretos. El primero hace referencia a los autores por sí mismos, analizando el momento en el que vivieron -auge, clímax y decadencia- y lo que es más importante, qué órdenes religiosas se van a adherir e impulsar las ideas de la escuela salmantina. En segundo lugar, la difusión por España y Europa de las obras de la Escuela de Salamanca. Éste es uno de los aspectos más desconocidos e interesantes del trabajo que estudiamos. Barrientos enumera para cada autor las ediciones de sus obras haciendo especial hincapié en la fecha y lugar de edición. Esta información nos permitirá reconstruir minuciosamente el impacto editorial de la Escuela de Salamanca en la Europa de la Edad Moderna, así como una recomposición del mapa de la influencia salmantina durante la Edad Moderna. En tercer lugar, un análisis sistemático de las citas que aparecen en la parte de sus tratados que dedican a cuestiones de moral económica, en total, un conjunto de 140.929 referencias que permite individuar quiénes constituyen en cada momento las autoridades intelectuales sobre las que asientan sus ideas, al tiempo que observamos cómo evoluciona en el tiempo la memoria colectiva de esta escuela. Para ello hemos tomado la fecha de la primera edición de la obra, que marca el momento de creación de una idea y es idóneo para reflejar el clima intelectual de la época. El estudio es de un enorme interés, pues el Renacimiento y el Humanismo impulsaron la revisión sistemática de las fuentes de la Tradición, al tiempo que la Reforma protestante discutía su autoridad, sentando las bases de un nuevo orden económico y social.

Por otro lado, el periodo de estudio está atravesado por la Reforma católica del Concilio de Trento, en el que van a participar algunos de nuestros teólogos. Surgen entonces las siguientes preguntas: ¿cómo influyó ese convulso y dinámico ambiente intelectual en las referencias que utilizan nuestros autores? ¿Hubo una vuelta a la Tradición, Patrística y Magisterio, tras el Concilio de

11 La recopilación de los datos, un minucioso trabajo de varios meses, fue realizada por Ludovico Mastrocinque, en su carácter de becario de la cátedra de Historia Moderna bajo la tutela de Antonio Moreno durante el curso académico 2011-2012. 
Trento? ¿Hubo un repliegue sobre los autores 'escolásticos', como fuente 'segura' en un momento de incertidumbre?

Respecto al análisis cuantitativo de los términos de referencia más importantes en el pensamiento de los autores analizados (Barrientos hace un inventario sistemático de los índices dedicados a temas de moral económica de cada una de las obras de los 95 autores que comenta) se analizan un total de 3.914 apartados. Los índices de las obras siempre están estructurados en varios niveles, por ejemplo: libro, cuestión, artículo, respuesta. Naturalmente, un libro puede tener varias cuestiones, cada cuestión puede tener varios artículos, y a su vez cada artículo puede tener varias respuestas. Por lo tanto, se ha empleado siempre como unidad de información el nivel mínimo, es decir el apartado menor (que, en este caso, sería la respuesta).

Esta metodología requiere aún una precisión. Lógicamente no es lo mismo que un término aparezca en un libro a que lo haga en una respuesta. Si el término aparece en el apartado menor no habría problema. La cuestión es cómo dar un peso mayor en nuestro análisis cuando un concepto aparece en apartados superiores, lo cual se resolvió calculando el producto de los apartados en los que el vocablo aparece. A modo de ejemplo: una obra con un libro que tiene 3 cuestiones, cada una de ellas con 3 artículos, y a su vez cada artículo tiene 5 respuestas. En ese caso, si el término aparece en el libro, su peso específico será igual a $3 * 3 * 5=45$; si aparece a nivel de cuestión, entonces sería $3 * 5=15$; mientras que, si aparece a nivel de artículo, entonces sería simplemente 5 . Se ha procedido de esta manera para medir la "importancia relativa" de cada uno de los 51 términos centrales escogidos como fiel imagen de los temas tratados por los autores.

Establecido el criterio cuantitativo para medir la importancia de los temas, se asigna su "presencia" nuevamente a la fecha de la primera edición de la obra. El objetivo del análisis es establecer las ideas centrales en torno a las que se desarrolla el pensamiento de la Escuela de Salamanca, su evolución en el tiempo -qué ideas pierden importancia con el paso del tiempo, qué otras nuevas les sustituyen- o cuál es el grado de asociación de las ideas entre sí. $\mathrm{O}$, por otro lado, cuáles son las ideas fuerza según las órdenes religiosas, para así mostrar las diferencias entre las distintas órdenes si las hubiera. Finalmente, cabe destacar que no es el objeto de este estudio describir el pensamiento de un autor, sino el lograr una aproximación a la atmósfera mental de una Escuela, describir el universo de referencias básicas en torno a las que organiza la resolución de los problemas económicos que se plantean. 


\section{ANÁLISIS DE AUTORES Y DIFUSIÓN DE SUS OBRAS}

En esta primera sección se intentará encontrar cómo se distribuyen temporal y espacialmente los autores de las dos categorías antes explicadas. Para ello, en primer lugar, se ha estudiado la evolución temporal del número de autores a lo largo de los siglos XVI y XVII por órdenes religiosas. Luego se verá la evolución temporal del número de ediciones que estos autores logran publicar y dónde lo hacen.

El gráfico 1 muestra el número de autores por orden religiosa. Para su elaboración, a la fecha de nacimiento de cada uno de los 95 autores se han añadido 35 años, considerando que es ése el umbral a partir del cual un autor es "fértil" literariamente y empieza a plasmar en su obra las influencias recibidas.

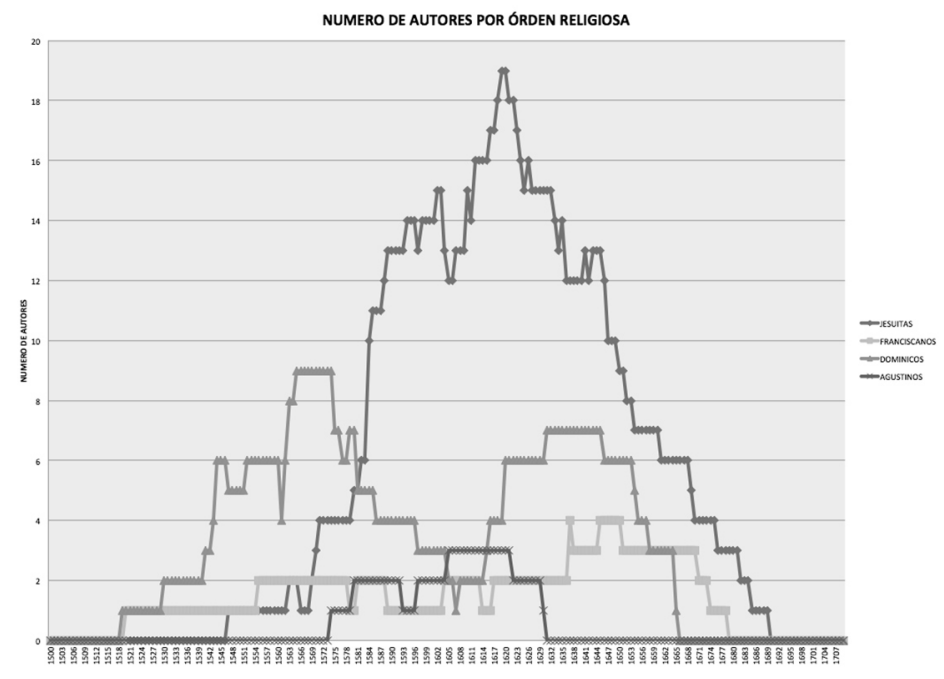

Gráfico 1. (Elaboración propia).

Como se ve en el gráfico, el número de autores no deja de crecer a lo largo del siglo XVI y comienzos del siglo XVII, llegando a un máximo en torno a los 20 primeros años del siglo XVII, para caer de modo precipitado en la segunda mitad de este siglo.

Asimismo, durante gran parte del siglo XVI franciscanos, agustinos y dominicos son las órdenes religiosas que aportan un mayor número de autores. En efecto, de los 10 maestros pertenecientes a la Escuela de Salamanca en sentido estricto la mayoría son dominicos (7 de 10), por lo que es perfectamente 
comprensible que los primeros en recoger y difundir sus enseñanzas sean, en su mayoría, miembros pertenecientes a la misma orden religiosa.

En el último cuarto del siglo XVI se produce, ya en el seno de la Proyección, un ascenso fulgurante de los autores pertenecientes a la Compañía de Jesús. El relevo entre jesuitas y dominicos se produce ya en 1581 (6 teólogos jesuitas contra 5 dominicos), quedando el devenir de la Proyección de la Escuela de Salamanca completamente en manos de los primeros.

De este primer análisis se puede resaltar la gran importancia e influencia que tenían los maestros de la Escuela de Salamanca, que en pocos decenios marcaron con sus obras a un gran número de autores. A pesar de ello no hay que olvidar, ni ahora ni a lo largo de los demás estudios empíricos que se llevarán a cabo, que los jesuitas eran -según Barrientos- los "nuevos teólogos que, bajo manto de seguidores de Santo Tomás, enseñaban doctrinas totalmente contrarias a las del doctor Angélico" ${ }^{12}$ al no ajustarse a las pautas del colegio de San Esteban para la interpretación del Aquinate.

El próximo paso, una vez analizado el número de autores, será determinar si existe una correspondencia entre éstos y el número de ediciones que publican. Que una orden religiosa posea un mayor número de autores no implica que tuviese una mayor influencia o importancia; ésta vendrá determinada por el número de ediciones. Para ello se ha elaborado la media móvil del número de ediciones que cada una de las órdenes vistas anteriormente publica a lo largo de los siglos XVI, XVII y XVIII, recogidas en el gráfico 2.

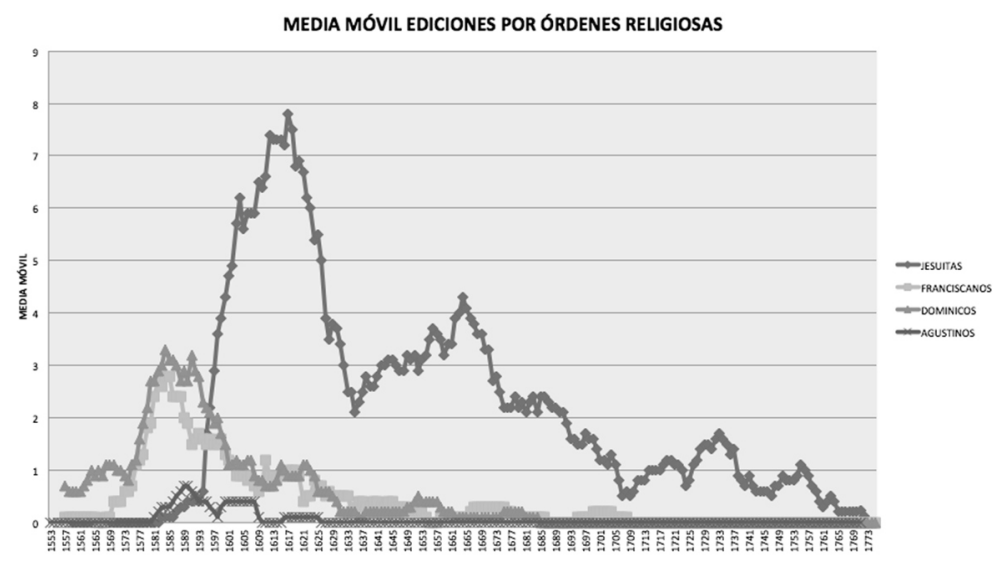

Gráfico 2. (Elaboración propia).

12 José Barrientos García, Repertorio de moral económica ..., 76. 
El resultado encaja bien con el análisis efectuado anteriormente. Durante gran parte del siglo XVI las ediciones pertenecientes a los miembros de las órdenes mendicantes (franciscanos y dominicos) son la mayoría, disminuyendo sensiblemente a partir de 1590, para ir cayendo lentamente en el olvido durante los dos siglos venideros.

Por su parte, las ediciones de los miembros de la Compañía de Jesús empiezan a hacerse sentir a partir de 1593, para protagonizar posteriormente un ascenso imparable, llegando a las 14 ediciones en 1622. A partir de entonces, la difusión de sus obras tiende a perder fuerza rápidamente. En la segunda mitad del siglo XVII el número de ediciones desciende a dos tercios, para casi desaparecer en el siglo XVIII, con apenas una obra por año como media. Es muy relevante resaltar que cuando aparece la Ilustración hacía ya varios decenios que el poder de difusión de las ideas de la Escuela de Salamanca y de su Proyección había decaído notablemente.

En cuanto a la difusión espacial de las ediciones abordadas vemos nuevamente fuertes diferencias entre órdenes religiosas. Las órdenes mendicantes centran sus ediciones en las ciudades españolas: Salamanca (38), Zaragoza (15), Barcelona (15), Madrid (12) y Toledo (10). A excepción de los casos de Lyon y de Venecia (34 y 16 ediciones respectivamente), las ediciones en las demás ciudades de Europa no supera nunca la decena. Por su parte, los jesuitas diseminan sus publicaciones a lo largo de todas las grandes urbes 'católicas' del Viejo continente, entre las que destacan Lyon (91), Venecia (55), Colonia (45), París (34), Amberes (35), Roma (27), Maguncia (15) y Milán (14). En cambio, su presencia en las ciudades españolas es escasa, e incluso nula, en ciudades como Salamanca y Toledo.

Para estudiar el peso relativo de las ediciones de cada orden religiosa en las mismas, y con el objetivo de facilitar el análisis y la comprensión de la gran cantidad de información que se está reflejando, se han agrupado los centros de publicación más importantes de España ${ }^{13} \mathrm{y}$ de Europa ${ }^{14}$, y se exponen en el Gráfico 3.

13 Las ciudades españolas con mayor número de ediciones son: Madrid, Barcelona, Salamanca, Toledo, Valladolid, Alcalá de Henares, Zaragoza, Valencia y Burgos.

14 Las ciudades europeas con mayor número de ediciones son: Lyon, Venecia, Colonia, Amberes, París, Roma, Milán, Madrid, Barcelona, Lisboa, Bruselas y Munich. 


\section{EDICIONES CIUDADES EUROPEAS POR ÓRDENES}

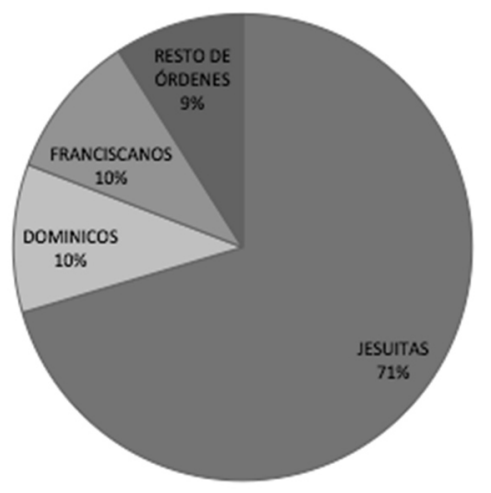

Gráfico 3 (Elaboración propia).

Como era de esperar, los jesuitas copan el 71\% de las ediciones en las grandes ciudades europeas de la época, mientras que la orden franciscana y la dominica aglutinan un exiguo $10 \%$ cada una. Si se pone la atención en las ciudades españolas, la imagen cambia diametralmente: en ellas los dominadores son los franciscanos (35\%) y los dominicos (30\%). Los jesuitas apenas publican un 17\% de sus ediciones en España. Los agustinos por su parte no alcanzan ni siquiera un $1 \%$ en ninguna de las categorías analizadas.

Con este primer análisis se concluye nada más allá de la existencia de una ruptura temporal y espacial en cuanto a las órdenes religiosas a las que pertenecen los teólogos estudiados. Se verá más adelante si las acusaciones que se vertían contra los jesuitas en el seno de la Universidad de Salamanca eran ciertas, ahondando en los temas y las citas que trataban en sus obras.

\section{ANÁLISIS DE CITAS E INFLUENCIAS}

El enorme volumen de citas plasmadas por Barrientos ha permitido dividir las citas que cada autor realiza en sus obras en siete categorías: Sagrada Escritura (Antiguo y Nuevo Testamento), Teólogos Medievales (de la Alta y Baja Edad Media), Magisterio y Padres de la Iglesia, Escuela de Salamanca, Teólogos 
de la Proyección, Canonistas y Civilistas y las Fuentes del Derecho (Derecho Romano, Canónico e Hispánico entre otros).

El objetivo es definir la 'memoria común' de la escuela; o al menos, comprobar si a lo largo del tiempo se mantienen referencias comunes fundamentales. El desarrollo temporal de algunas de las citas más relevantes ayudará a dilucidar si los teólogos de la Proyección comparten las mismas fuentes que los de la Escuela de Salamanca. Aunque ya se ha visto que pertenecen eminentemente a órdenes religiosas distintas de las anteriores y que publican en lugares distintos, lo que se pretende ahora es ver si son verídicas las acusaciones vertidas contra los jesuitas, considerados como "nuevos teólogos" 15 , rompedores de la tradición anterior. Para ello, el primer análisis no puede ser otro que comparar la evolución temporal de las citas a teólogos medievales (donde se encuentra Santo Tomás de Aquino, figura primordial entre todas, junto a autores como Silvestre Oreiro, Tomás de Vio Cayetano, Duns Escoto, y Conrado de Summerhat, entre otros) con las citas que los miembros de la Proyección realizan de sí mismos.

La hipótesis que aquí se sostiene es que, si estas acusaciones vertidas contra la Compañía de Jesús fueran falsas, el peso de las citas hechas a los teólogos medievales, en especial al Aquinate, debería mantenerse constante a lo largo del tiempo. Para saber qué ocurrió se utiliza el número de citas recabado por Barrientos (en porcentaje del total de citas del periodo) para cada categoría estudiada.

El Concilio de Trento - finalizado en 1563- provoca un trastoque inicial de las referencias de los autores, como se puede apreciar en la fuerte caída (casi un 15\%) que conduce al punto relativo al decenio 1570-1579. Los teólogos medievales representaban hasta entonces casi la mitad de las citas, siendo para los autores la referencia intelectual más relevante para solucionar las cuestiones de moral económica planteadas. Es entonces cuando se produce un relevo en dichas referencias, pasando los teólogos de la Proyección a citarse masivamente entre sí. A finales del siglo XVII el $80 \%$ de las citas son a teólogos de la Proyección, mientras que los medievales apenas llegan al 10\% (ver Gráfico 4). Así, los datos sugieren un giro importante en la 'memoria común' como referencia de su pensamiento.

15 José Barrientos García, Repertorio de moral económica ..., 201. 


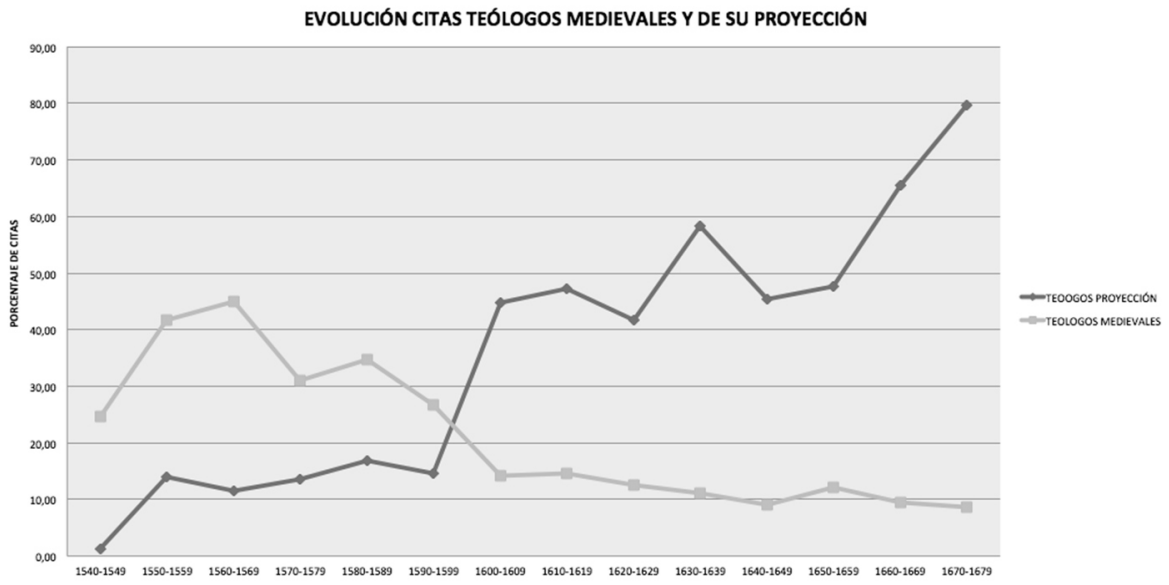

Gráfico 4 (Elaboración propia).

Según nuestro análisis de los datos que aporta Barrientos, la evolución de las citas relativas a Santo Tomás de Aquino sufre una evolución similar. A mediados del siglo XVI, es el autor individual más citado en las obras de moral económica analizadas; tras Trento, su importancia se diluye progresivamente, representando apenas el 2-3\% de las citas en las obras del siglo XVI (ver Gráfico 5).

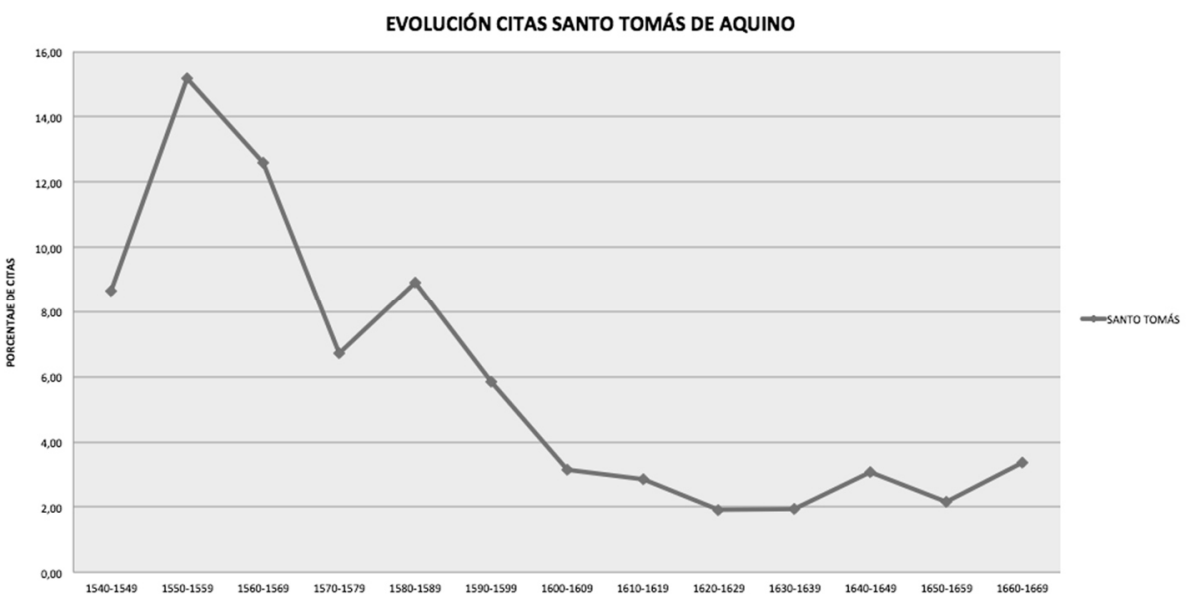

Gráfico 5 (Elaboración propia). 
Según los datos de Barrientos, la evolución de las citas de la Sagrada Escritura, Patrística y Magisterio sufren una evolución distinta, marcada por los conflictos en el seno de la Universidad de Salamanca y por las disputas entre biblistas y escolásticos en Trento, que reflejan la existencia de dos tendencias encontradas en el seno de la Universidad. Según Barrientos, la primera, anclada en el escolasticismo especulativo, tenía una mentalidad estrecha, rutinaria y estática; miraba al pasado y se oponía a toda innovación y a un sano y prudente progreso. La otra, más abierta y dinámica, cree en un prudente progreso y trata de conciliar lo positivo y lo especulativo, dando cabida a las exigencias de los humanistas y utilizando los avances de la filología y los conocimientos lingüísticos en la interpretación de los textos bíblicos al momento de explicar el dogma. Esta tendencia no despreciaba la escolástica -aunque de ello fuera acusada-; antes la creía útil y necesaria, pero dentro de unos justos límites ${ }^{16}$.

Los máximos exponentes de la primera corriente -denominada "tradicionalista"- eran Cano, Sotomayor, Peña, Gallo, Guevara, Medina y, sobre todo, Báñez ${ }^{17}$. Estos rompieron las vinculaciones con el humanismo abiertas por Vitoria y continuadas por Soto y $\mathrm{Cano}^{18}$, iniciando un proceso de análisis y rectificación de la doctrina. Precisamente cuando dicho proceso entraba en plena ebullición aparecieron los denominados "hebraístas", pertenecientes a la segunda corriente: Fray Luis de León, Gaspar de Grajal y Martín Martínez de Cantalapiedra, quienes, como se ha dicho, buscaron incorporar a los métodos de interpretación de la Sagrada Escritura las conquistas y aportaciones de la lingüística y del estudio de las letras antiguas ${ }^{19}$.

En efecto los tradicionalistas, defendiendo el valor de la escolástica ${ }^{20}$, acabaron por aplastar a la corriente progresista, ya de suyo minoritaria, con la investigación inquisitorial y procesamiento de los tres maestros hebraístas (1571$1578)^{21}$. El estudio cuantitativo de las citas realizado confirmaría estas tendencias históricas apuntadas. El proceso de "rectificación" obrado por algunos de los maestros salmantinos, distanciándose de las corrientes humanistas abiertas por Vitoria, se puede apreciar a través de la fuerte caída que experimentan las citas a la Sagrada Escritura, al Magisterio de la Iglesia y a la Patrística durante el decenio 1550-1559, perdiendo gran parte de su peso.

16 Ibidem, 58.

17 Ibidem.

18 Vicente Beltrán Heredia, “Accidentada y efímera aparición del nominalismo...”, 525.

19 José Barrientos García, Repertorio de moral económica ..., 58.

20 Ibidem, 80.

21 Ibidem, 83. 
La práctica desaparición de la corriente progresista tras el proceso inquisitorial dará inicio a una tendencia que será continuada por los teólogos de la Proyección a lo largo del XVII. Los teólogos jesuitas, a diferencia de lo que ocurría antes, prosiguen con este "legado" intelectual de sus maestros y se alejan todavía más de las fuentes originales.

Por último, dada la tremenda importancia que tendrá el derecho (ius) a lo largo de los siglos XVI y XVII ${ }^{22}$, se analiza la evolución temporal de la importancia de las referencias por parte de los autores de la Escuela al Derecho Romano y al Derecho Canónico, en el Gráfico 6.

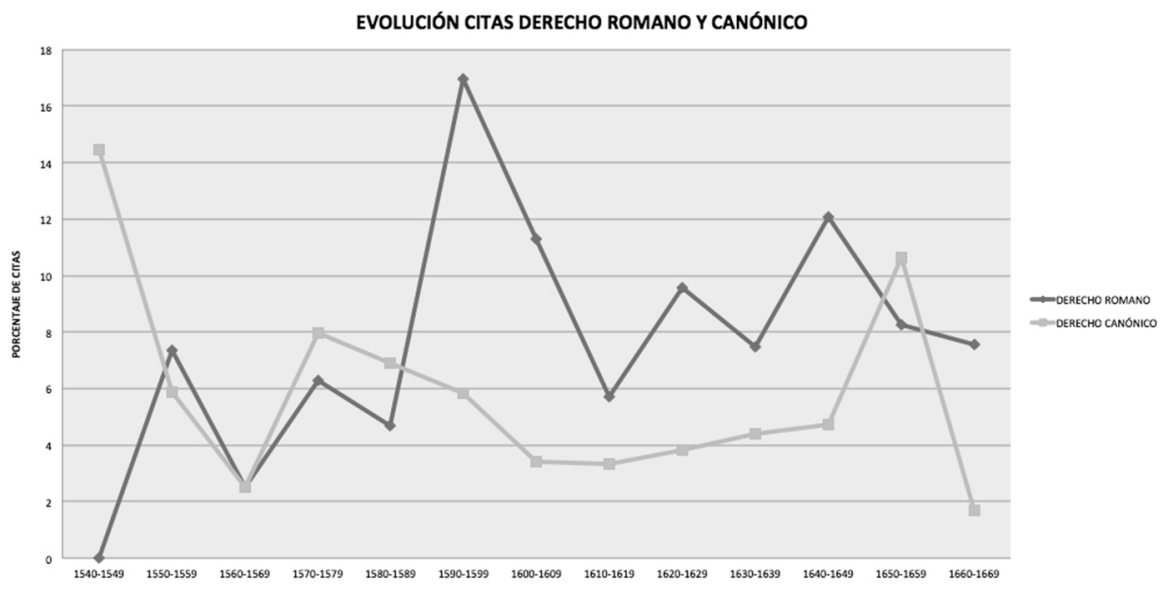

Gráfico 6 (Elaboración propia).

Según los datos que aporta Barrientos, para los teólogos de la Escuela de Salamanca el Derecho Canónico posee, como fuente de conocimiento para la resolución de problemas, una importancia mucho mayor a la que tiene el Derecho Romano. Esta tendencia se modifica suavemente ya a finales del siglo XVI, cuando a la importancia del Derecho Canónico se añade un creciente interés por el Derecho Romano, que es fuente mayoritaria de referencia a partir de 1590. Ello, como se verá a continuación, está en sintonía con la evolución temporal de las preocupaciones de los teólogos, centradas en el XVI y en el XVII en temas de naturaleza "financiera".

22 Para el auge de las universidades en estos siglos y de las facultades de derecho, véase Richard L. Kagan, Universidad y sociedad en la España moderna (Madrid: Tecnos, 1981). 


\section{ANÁLISIS TEMÁTICO}

Para una aproximación a los contenidos fundamentales de la Escuela de Salamanca, hemos analizado la frecuencia de todos los términos utilizados en los índices de las 95 obras reunidas en la base de datos. De estos términos, hemos seleccionado 54 conceptos, de acuerdo a las palabras con frecuencias más altas, pero también teniendo en cuenta aquellos términos que, por el espíritu de la Escuela, cabría esperar, como por ejemplo conceptos ligados a la amistad, la caridad, el don, o bien a los radicales económicos ${ }^{23}$. Esta primera aproximación nos permitió un acercamiento a las ideas centrales que vertebran el discurso de los salmantinos, así como sus intereses, de acuerdo con los temas presentes -o ausentes- en sus tratados, que, en un segundo momento, hemos agrupado en grandes áreas temáticas, cómo veremos a continuación.

Conviene recordar que la Escuela de Salamanca se enfrenta a un momento histórico de gran relevancia,

(...) un momento crucial de cambios: a nivel cultural -auge del humanismo, económico -creación de un marco económico moderno-, político -surge el estado moderno, separación de los poderes de la Iglesia y el Estado-, social -descubrimiento de América-, eclesial -reforma protestante y reacción católica, el Concilio de Trento-, teológico (.... ${ }^{24}$

En este contexto, la perspectiva medieval, fundamentada en el planteamiento aristotélico del dinero como medio de cambio y estéril en una economía estática, resultaba ineficaz para hacer frente a las revoluciones comercial y de precios que transformaron la economía y la sociedad del siglo $\mathrm{XVI}^{25}$, haciéndose cada vez más insostenible ${ }^{26}$.

Los autores salmantinos realizan un esfuerzo inusitado para conciliar la perspectiva clásica con estos cambios, manteniéndose fieles al espíritu doctrinal: en ningún momento suprimen la prohibición de la usura, basada en la

23 Lógicamente, hemos eliminado artículos, preposiciones, adverbios, así como términos que expresen el carácter de un apartado de una obra (tratado, libro, cuestión, artículo...) para referirnos únicamente a los conceptos que hacen referencia al contenido doctrinal de la escuela. También hemos reunido como un mismo concepto todos aquéllos derivados con igual significado, por ejemplo, para el caso de contrato: contractus, contracta, contractibus, contractu, contractui, contractum, contractuum, contrato, contratos. En este mismo sentido, cabe mencionar además que se ha realizado la búsqueda tanto en latín como en español, unificando los significados.

24 Idoya Zorroza, Francisco de Vitoria. Contratos y usura (Pamplona: EUNSA, 2006), 45.

25 Véase Enrique Fuentes Quintana. Economía y economistas españoles I (Barcelona: Galaxia Gutenberg, 1999), 44-45.

26 Teodoro López. Mancio y Bartolomé de Medina: Tratado sobre la usura y los cambios (Pamplona: EUNSA, 1998), 11. 
ilicitud de vender por separado la sustancia y el uso en el caso de bienes fungibles -es decir, de aquéllos cuyo uso es su consumo, como es el caso del dinero${ }^{27}$. No obstante, se aceptan ciertos 'títulos extrínsecos' según los cuáles es lícito recibir cierto lucro; el más común es el damnum emergens (daño emergente) relacionado con los riesgos asumidos al prestar; pero también están el lucrum cessans (lucro cesante), el periculum sortis (o riesgo de incobranza), la poena conventionalis, por demoras en la devolución del dinero prestado, o el titulus legis civilis, o amparo de legitimidad en un interés fijado por ley. Al hablar de lucro, dominan la reflexión sobre el lucro cesante y el daño emergente, que desde 1600 adquieren prácticamente la misma importancia.

Desde el punto de vista cuantitativo, destaca el término contractus y sus derivados (1610 veces); seguido por venta (1036), iustitia (959), emptio (944) y usura (880). Con frecuencia superior a 400, aparecen los términos licet (lícito, 720) duda (710), mutuo (633), cambii (412), iure (457) y pretium (448). Sorprende la centralidad del término 'contrato' en una Escuela considerada 'escolástica' y, por lo tanto, anclada en la Baja Edad Media, lo cual lleva a pensar que la importancia del contrato como arquetipo de las relaciones sociales hunde su origen en la escolástica bajomedieval, reafirmándose claramente durante los siglos XVI y XVII en la Escuela de Salamanca. Sin embargo, conviene destacar qué tipo de contrato se reafirma como arquetipo en dicha Escuela. Si unimos bajo una misma categoría 'venta' y 'emptio' (1980 veces) se intuye que los autores salmantinos están pensando en la relación de compraventa como arquetipo de la relación contractual propia del mundo mercantil.

En efecto, de los radicales de la economía -propiedad, moneda y trabajodestaca sobremanera el tema del dinero, a mucha distancia del tema de la propiedad. En torno a la propiedad, los temas centrales son: dominium, possesio, propietas, usufructum, emphyteusis, feudo, donatio, census y arrendamiento; respecto al dinero, destacan: usura, intereses, préstamo, pecunia, lucrum, cambii, census; y el tema del trabajo es prácticamente irrelevante, mencionando: industria, labor, officium, negotium y opus. Sorprende la escasa importancia del término propiedad, tal como cabría esperar en la era de la Aristocracia. Respecto a este concepto, cabe destacar que, según los datos aportados por Barrientos, la centralidad del término 'dominium' para referirse a la propiedad comienza a ser reemplazado por 'possesio' y 'propietas' desde comienzos del siglo XVII, en paralelo con lo que sucede en el resto de Europa durante la modernidad.

27 Véase Idoya Zorroza, op. cit., 58, y José Barrientos, Un siglo de moral económica en Salamanca (Salamanca: Universidad de Salamanca, 1985), 77 y ss. 


\section{CONCLUSIONES}

El análisis de los datos recopilado por Barrientos ofrece una aproximación a la evolución del pensamiento económico a lo largo de más de tres siglos, en un momento histórico clave caracterizado por un cambio paradigmático sin precedentes. La Escuela de Salamanca representa una verdadera "bisagra" entre la postura clásica aristotélico-tomista y la vía moderna en materia social y económica, a la vez que un esfuerzo patente por mantener el espíritu tomista en que se inspira, en una época de cambios considerables. Se observan en la evolución temporal de la Escuela cambios significativos en cuanto a ediciones, lugares de publicación, citas y preocupaciones sociales, lo cual implica, desde un punto de vista empírico, el reforzamiento de aquellas teorías que proclaman una división entre los miembros de la Escuela y aquéllos que son influenciados por éstos. Sin duda, el análisis de los datos de Barrientos muestra cómo la Escuela de Salamanca llegó a tener un amplio eco al menos en la Europa católica entre 1590 y 1640 .

Aunque durante gran parte del siglo XVI las órdenes religiosas que destacan son franciscanos, agustinos y dominicos, a finales del siglo XVI adquieren mayor preponderancia los autores pertenecientes a la Compañía de Jesús, pasando a ser mayoría en 1581, y dominando la proyección de la Escuela desde finales del siglo XVI, muchas veces con doctrinas que empiezan a distanciarse del tomismo. En el siglo XVII los jesuitas tienen prácticamente el monopolio de las ediciones en las grandes ciudades europeas de la época. Así, la sustitución de los mendicantes por los jesuitas parece asociada a un cambio en la memoria del grupo - menos referencias 'medievales' y 'patrísticas', más referencias 'modernas'-, lo que sugiere una cierta ruptura temporal y espacial en la escuela, que difícilmente se puede calificar como una 'vuelta al pasado escolástico' sin más. Del mismo modo, por los temas tratados, se ve que trata de enfrentarse a y resolver los nuevos problemas más acuciantes del momento.

Si bien para los teólogos de la Escuela de Salamanca el derecho canónico posee una gran importancia para la resolución de problemas, el estudio de las citas sugiere desde finales del siglo XVI una creciente relevancia del derecho romano civil. La justicia es claramente el marco de referencia fundamental para valorar las acciones que consideran estos autores. Aunque en el dilema ius-iustitia, vence iustitia, el gran cambio a lo largo del desarrollo de la Escuela es la creciente importancia del ius. Al menos, la evolución en el tiempo de las referencias utilizadas por estos autores sugiere una importancia cada vez mayor del derecho, especialmente del civil romano. 
Posiblemente, esta creciente "juridificación" esté en sintonía con las preocupaciones de los teólogos de la proyección del siglo XVI y XVII en temas de naturaleza "financiera". Precisamente, los temas a que darán mayor importancia serán aquéllos ligados a la moneda o financieros, así como los asociados al contrato y las compraventas. El dominio de los problemas financieros, ligados a la moneda, permite hablar de una doctrina que se enfrenta a los problemas propios de un mundo urbano, altamente monetizado y donde el comercio es de una importancia central.

Por último, uno de los datos más sólidos de este trabajo es el enorme número de referencias dentro de la Escuela al contrato, sin duda uno de los rasgos centrales de la modernidad. Observamos dos asociaciones intensas. Por un lado, la frecuente relación entre el término contrato y las palabras compra o venta. Sin duda, el núcleo de la reflexión se está refiriendo al nuevo mundo mercantil. Por otro lado, la relación entre contrato y justicia. ¿Qué justicia? Sin duda la justicia conmutativa, 'la propia de los contratos' como dicen ellos, pero también la justicia distributiva. En este sentido, la escuela de Salamanca es ya una escuela 'moderna' por las preocupaciones a las que se enfrenta, aunque no por 'cómo' se enfrenta a ellas.

En efecto, si hay algo que caracteriza a la Edad Moderna es el intento de construir una sociedad sobre la base del contrato -la relación contractual-, como se muestra en pensadores modernos como Hobbes, Locke, Rousseau, entre otros. Estos autores -llamados precisamente contractualistas- consideran que, en la entraña de la sociedad, fundándola, no está el don sino la relación contractual. Lo que este estudio demuestra es que este proyecto social tiene un precedente en la Escuela de Salamanca, de inspiración tomista, afirmándose claramente durante los siglos XVI y XVII, lo que posiblemente obliga a desplazar su origen en el tiempo hasta la Baja Edad Media. Esto podría ayudar a comprender mejor la génesis de la teoría económica moderna, así como el desarrollo de la historia política de Occidente.

Así, este trabajo muestra que ya en la Escuela de Salamanca el contrato es un tema central, tal como plantea el mundo moderno. Por ello, sugerimos que el estudio sistemático de las semejanzas y diferencias en la comprensión del contrato entre los salmantinos y los autores posteriores puede abrir una vía privilegiada para la comprensión de las relaciones entre el mundo medieval, el humanismo renacentista y la modernidad.

Se ha dicho que el mundo moderno nace cuando se acepta que todo (patrimonio, trabajo, etc.) es medible por el dinero, y, éste, es una mercancía más, como el resto de las mercancías: su precio varía con la oferta y la demanda. Así, 
en la comprensión capitalista de los cosas, tierra, trabajo y dinero son mercancías $^{28}$. En ese universo es muy difícil conciliar la relación contractual y la lógica del don.

Sin embargo, en la comprensión del dinero de la primera escolástica, el dinero es un don ${ }^{29}$, funda relaciones de amistad, lo que es el fundamento antropológico de la inmoralidad de la usura. Frente a ello, la Escuela de Salamanca ha empezado a defender la teoría cuantitativa del dinero -su valor varía en función de su 'abundancia' o 'carestía'-, lo que sugiere que, en ciertos contextos -entre naciones y en el comercio internacional-, para estos autores la moneda se comporta como una mercancía. ¿Cómo afectó esta doble comprensión de la moneda a su visión de las relaciones contractuales?, especialmente, de los contratos mercantiles y del préstamo de dinero. Fiel a la tradición escolástica, la escuela salmantina sigue manteniendo la necesidad de conciliar en los contratos la justicia conmutativa con la gratuidad propia del don, expresión de la amistad ${ }^{30}$. Pero sin duda su nueva comprensión del dinero podría dejar sin fundamento antropológico la prohibición de la usura y sin espacio social a las relaciones regidas por la justicia distributiva, fundamento de su comprensión estamental de la sociedad.

\section{REFERENCIAS BIBLIOGRÁFICAS}

Barrientos García, José. Repertorio de moral económica (1536-1570): La Escuela de Salamanca y su proyección. Pamplona: EUNSA, 2011.

- Un siglo de moral económica en Salamanca. Salamanca: Universidad de Salamanca, 1985.

Belda Plans, Juan. "Hacia una noción crítica de la Escuela de Salamanca", Scripta Theologica 31 (1999): 367-411.

28 Ésa es la tesis central de Karl Polanyi en La Gran Transformación. Crítica del liberalismo económico (Madrid: Ediciones La Piqueta, 1947).

29 Para el dinero como don en la escolástica bajomedieval, véase John T. Jr. Noonan, The Scholastic Analysis of Usury, (Cambridge, Massachusetts: Harvard University Press, 1957), 104-105.

Según Noonan esta opinión -la del dinero como don que funda relaciones personales de amistades ampliamente aceptada -al menos por Guillermo de Auxerra, Raimundo de Peñafort, el Hostiensis, San Buenaventura, Bernardino de Siena y Antonio de Florencia-, y de un alcance social grande en la época, pues son figuras principales de la escolástica.

Así, Bernardino de Siena (1380-1444), al criticar la usura dice que "la usura concentra el dinero de la comunidad en manos de unos pocos, como si toda la sangre del cuerpo de un hombre fuera a la cabeza y dejara los otros órganos agotados" Citado en , John T. Jr. Noonan The Scholastic ... 74 (La negrita es nuestra).

Así, la metáfora del dinero como 'sangre' del cuerpo social -por tanto 'alma' que da vida a la comunidad- refrenda la comprensión del dinero como don de la vida social de la comunidad.

30 Esta es la tesis principal de Bartolomé Clavero, Antidora. Antropología Católica de la Economía Moderna (Milán: Giuffrè Editore, 1991). 
Beltrán de Heredia, Vicente. “Accidentada y efímera aparición del nominalismo en Salamanca". En Miscelánea de Beltrán de Heredia: Colección de artículos sobre la historia de la teología española. Salamanca, 1972.

Clavero, Bartolomé. Antidora. Antropología Católica de la Economía Moderna. Milán: Giuffrè Editore, 1991.

Fuentes Quintana, Enrique. Economía y economistas españoles I. Barcelona: Galaxia Gutenberg, 1999.

Gómez Rivas, León. "Business Ethics and The History of Economics in Spain «The School of Salamanca: A Bibliography»", Journal of Business Ethics 22 (1999): 191-202.

López, Teodoro. Mancio y Bartolomé de Medina: Tratado sobre la usura y los cambios. Pamplona: EUNSA, 1998.

Muñoz Delgado, Vicente. Lógica, ciencia y humanismo en la renovación teológica de Vitoria y Cano. Madrid: Instituto Francisco Suárez del C.S.I.C, 1980.

Noonan, John T. Jr. The Scholastic Analysis of Usury. Cambridge MA: Harvard University Press, 1957.

Polanyi, Karl. La Gran Transformación. Crítica del liberalismo económico. Madrid: Ediciones La Piqueta, 1947.

Zorroza, Idoya. Francisco de Vitoria. Contratos y usura. Pamplona: EUNSA, 2006.

Germán Scalzo

Facultad de Ciencias Económicas y Empresariales

Universidad Panamericana Augusto Rodin 498, Insurgentes Mixcoac 03920 Ciudad de México (México) https://orcid.org/0000-0002-8229-713X

Antonio Moreno Almárcegui

Facultad de Económicas

Universidad de Navarra

Edificio Amigos, Campus Universitario

31009, Pamplona, Navarra (España)

Orcid: https://orcid.org/0000-0003-4725-0325. 
